\title{
The Homotopy Analysis Method for Approximating of Giving Up Smoking Model in Fractional Order
}

\author{
Anwar Zeb ${ }^{1}$, M. Ikhlaq Chohan², Gul Zaman¹ \\ ${ }^{1}$ Department of Mathematics, University of Malakand, Khyber Pakhtunkhawa, Pakistan \\ ${ }^{2}$ Department of Business Administration and Accounting, Buraimi University College, Al-Buraimi, Oman \\ Email: gzaman@uom.edu.pk
}

Received June 6, 2012; revised July 6, 2012; accepted July 14, 2012

\begin{abstract}
In this paper, we consider the giving up smoking model. First, we present the giving up smoking model in fractional order. Then the homotopy analysis method (HAM) is employed to compute an approximate and analytical solution of the model in fractional order. The obtained results are compared with those obtained by forth order Runge-Kutta method and nonstandard numerical method in the integer case. Finally, we present some numerical results.
\end{abstract}

Keywords: Fractional Differential Equations; Epidemic Model; Homotopy Analysis Method

\section{Introduction}

The common Calculus has been studied well and its applications can be encountered in several areas of science and engineering. Relating to fractional Calculus, it is not familiar to several researchers. Indeed, fractional Calculus is a three centuries old mathematical tools. But the searching of the theory of differential Equations of fractional order has just been began quite recently [1-3]. An expanding of fractional notions in Biomathematics has also been improved. Fairly, no field of standard analysis has been left unconcerned by fractional Calculus. Smoking is one of the most important health problems in the world and it infentend different organ of human body which cover many death in all over the world. Smoking is dangerous to people health even only for a short term period. The effects of short smoking are bad breath, stained teeth, smell of smoke in the fingers and hair. Other effects on a temporary basis are also coughing, rapid heart rate, high blood pressure and sore throat. The long term effects of smoking are considered more threatening and these are lung cancer, throat cancer, mouth cancer and gum disease, heart disease, stomach ulcers, emphysema and other smoke related conditions. In fact, because of the nature of the long term effects, millions of people around the world have already died from smoking. All of these matters can be stopped if they are treated. Another way is merely to abdicate cigarettes. The aim of this paper is to enhance two numerical schemes for solving a mathematical model describing a giving up smoking model and shows the dynamical interaction.

There has been some efforts made in the mathematical modeling of giving up smoking since the 2000s. In [4], Zaman proposed a modified model that describes giving up smoking model. In his paper he studied the qualitative behavier of smoking and represented numerical simulation by using numerical methods. The homotopy analysis method (HAM) is proposed first by Liao [5,6] for solving linear and nonlinear differential and integral equations. Different from perturbation techniques; the (HAM) doesn't depend upon any small or large parameter. This method has been successfully applied to solve many types of nonlinear [5-10] differential equations. In this paper, (HAM) is applied to solve nonlinear fractional initialvalue problem of the non-fatal epidemic model to obtain symbolic approximate solutions for linear and nonlinear differential Equations of fractional order. (HAM) is different from all analytical methods; it provides us with a simple way to adjust and control the convergence region of the series solution by introducing the auxiliary parameter $h$ and the auxiliary function. In fact, it is the auxiliary parameter $h$ that provides us, for the first time, a simple way to ensure the convergence of the series solution. Due to this reason, it seems reasonable to rename $h$ the convergence-control parameter. It should be emphasized that, without the use of the convergence-parameter, one had to assume that the homotopy series is convergent. However, with the use of the convergence-parameter $h$, such an assumption is unnecessary; because it seems that one can always choose a proper value of $h$ to obtain convergent homotopy-series solution. So, the use of the convergence-parameter $\mathrm{h}$ in the zeroth-order deformation equation greatly modifies the early homotopy analysis method. Since then, the homotopy analysis method has 
been developing greatly and more generalized zerothorder deformation Equations are suggested by Liao [5,6]. The adomain decomposition method (ADM), the homotopy perturbation method (HPM), the variational iteration method (VIM) some other numerical methods have been used to provide analytical approximation to linear and nonlinear problems. In this paper we apply the HAM to compute the approximate solution of the proposed model.

This paper is organised as: In Section 2, we present formulation of the model with some basic definitions and notations related to this work. In Section 3, the homotopy analysis method (HAM) is applied to the model. In Section 4, the numerical simulations are presented graphically. In Section 5, we give conclusion. Finally, we give acknowledgment.

\section{Preliminaries and Formulation of Model}

In this section we present some basic definitions which are necessary for the subsequent sections. A function $f(x)(x>0)$ is said to be in the space $C_{\alpha}(\alpha \in R)$ if it can be written as $f(x)=x^{p} f_{1}(x)$ for some $p>\alpha$ where $f_{1}(x)$ is continuous in $[0, \infty)$, and it is said to be in the space $C_{\alpha}^{m}$ if $f^{(m)} \in C_{\alpha}, m \in N$.

The Riemann-Liouville integral operator of order $\alpha>0$ with $a \geq 0$ is defined as

$$
\begin{aligned}
& \left(J_{a}^{\alpha} f\right)(x)=\frac{1}{\Gamma(\alpha)} \int_{a}^{x}(x-t)^{\alpha-1} f(t) \mathrm{d} t, x>a, \\
& \left(J_{a}^{\alpha} f\right)(x)=f(x),
\end{aligned}
$$

We only need here the following:

For $f \in C_{\alpha}, \alpha, \beta>0, a \geq 0, c \in R$ and $\gamma>-1$, we have

$$
\begin{aligned}
& \left(J_{a}^{\alpha} J_{a}^{\beta} f\right)(x)=\left(J_{a}^{\beta} J_{a}^{\alpha} f\right)(x)=\left(J_{a}^{\alpha+\beta} f\right)(x), \\
& J_{a}^{\alpha} x^{\gamma}=\frac{x^{\gamma+\alpha}}{\Gamma(\alpha)} B_{\frac{x-a}{x}}(\alpha, \gamma+1) .
\end{aligned}
$$

where $B_{\tau}(\alpha, \gamma+1)$ is the incomplete beta function which is defined as

$$
\begin{gathered}
B_{\tau}(\alpha, \gamma+1)=\int_{0}^{\tau} t^{\alpha-1}(1-t)^{\gamma} \mathrm{d} t \\
J_{a}^{\alpha} e^{c x}=e^{a c}(x-a)^{\alpha} \sum_{k=0}^{\infty} \frac{[c(x-a)]^{k}}{\Gamma(\alpha+k+1)}
\end{gathered}
$$

The Riemann-Liouville derivative has certain disadvantages when trying to model real-world phenomena with fractional differential equations.

The Caputo fractional derivative of $f(x)$ of order $\alpha>0$ with $a \geq 0$ is defined as

$$
\begin{aligned}
\left(D_{a}^{\alpha} f\right)(x) & =\left(J_{a}^{m-\alpha} f^{(m)}\right)(x) \\
& =\frac{1}{\Gamma(m-\alpha)} \int_{a}^{x} \frac{f^{(m)}(t)}{(x-t)^{\alpha+1-m}} \mathrm{~d} t,
\end{aligned}
$$

for $m-1<\alpha \leq m, m \in N, x \geq a, f(x) \in C_{-1}^{m}$.

The Caputo fractional derivative was investigated by many authors, for $m-1<\alpha \leq m, f(x) \in C_{\alpha}^{m}$ and $\alpha \geq-1$, we have

$$
\begin{aligned}
\left(J_{a}^{\alpha} D_{a}^{\alpha} f\right)(x) & =J^{m} D^{m} f(x) \\
& =f(x)-\sum_{k=0}^{m-1} f^{(k)}(a) \frac{(x-a)^{k}}{k !} .
\end{aligned}
$$

The definition of fractional derivative involves an integration which is non-local operator (as it is defined on an interval) so fractional derivative is a non-local operator. In other word, calculating time-fractional derivative of a function $f(t)$ at some $t=t_{1}$ time requires all the previous history, i.e. all $f(t)$ from $t=0$ to $t=t_{1}$.

Now we introduce fractional order into the giving up smoking model presented by Zaman by replacing the first time derivative term by a fractional derivative of order $\alpha_{1}, \alpha_{2}, \alpha_{3}, \alpha_{4}, \alpha_{5}>0$. The new system is described by the following system of fractional order differential equations:

$$
\begin{aligned}
D_{t}^{\alpha_{1}} P(t)= & b N(t)-\beta_{1}(t) L(t) P(t) \\
& -\left(d_{1}+\mu\right) P(t)+\tau Q(t), \\
D_{t}^{\alpha_{2}} L(t)= & \beta_{1} L(t) P(t)-\beta_{2}(t) L(t) S(t) \\
& -\left(d_{2}+\mu\right) L(t), \\
D_{t}^{\alpha_{3}} S(t)= & \beta_{2} L(t) S(t)-\left(\gamma+d_{3}+\mu\right) S(t), \\
D_{t}^{\alpha_{4}} Q(t)= & \gamma S(t)-\left(\tau+d_{4}+\mu\right) Q(t),
\end{aligned}
$$

By adding (1)-(4), we have

$$
\begin{aligned}
D_{t}^{\alpha_{5}} N(t)= & (b-\mu) N(t) \\
& -\left(d_{1} P(t)+d_{2} L(t)+d_{3} S(t)+d_{4} Q(t)\right) .
\end{aligned}
$$

where $N(t)=P(t)+L(t)+S(t)+Q(t)$.

Under the initial conditions:

$$
\begin{aligned}
& P(0)=c_{1}, L(0)=c_{2}, S(0)=c_{3}, \\
& Q(0)=c_{4}, N(0)=c_{5},
\end{aligned}
$$

where $P(t), L(t), S(t), Q(t)$ and $N(t)$ denote the numbers of potential smokers, occasional smokers, smokers, quit smokers and total smokers at time $t$, respectively. Here $b$ is the birth rate, $\mu$ is the natural death rate, $\gamma$ is the recover rate from smoking, $\beta_{1}$ and $\beta_{2}$ are transmission coefficients, $d_{1}, d_{2}, d_{3}$ and $d_{4}$ represent the death rate of potential smoker, occasional smoker, smoker 
and quit smokers, respectively. Additionally, $\tau$ represents the rate at which the quit smoker in the population becomes potential smoker again.

For $\alpha_{1}=\alpha_{2}=\alpha_{3}=\alpha_{4}=\alpha_{5}=\alpha$ where $0<\alpha<1$ this system is reduced to the model presented by Shaher et al. [11] as

$$
\begin{aligned}
D_{t}^{\alpha} P(t)= & b N(t)-\beta_{1}(t) L(t) P(t) \\
& -\left(d_{1}+\mu\right) P(t)+\tau Q(t), \\
D_{t}^{\alpha} L(t)= & \beta_{1} L(t) P(t)-\beta_{2}(t) L(t) S(t) \\
& -\left(d_{2}+\mu\right) L(t), \\
D_{t}^{\alpha} S(t)= & \beta_{2} L(t) S(t)-\left(\gamma+d_{3}+\mu\right)(t), \\
D_{t}^{\alpha} Q(t)= & \gamma S(t)-\left(\tau+d_{4}+\mu\right) Q(t), \\
D_{t}^{\alpha} N(t)= & (b-\mu) N(t) \\
& -\left(d_{1} P(t)+d_{2} L(t)+d_{3} S(t)+d_{4} Q(t)\right),
\end{aligned}
$$

where $D_{t}^{\alpha}$ is fractional derivative in the Caputo sense and $\alpha$ is a parameter describing the order of the fractional time-derivative with $0<\alpha<1$, subject to the same initial conditions

$$
P(0)=c_{1}, L(0)=c_{2}, S(0)=c_{3}, Q(0)=c_{4}, N(0)=c_{5} \text {. }
$$

\section{Homotopy Analysis Method (HAM)}

We apply the homotopy analysis method to find an approximate solution of Equations (1)-(5), which gives an accurate solution over a longer time frame as compared to the standard homotopy analysis method (HAM). For this purpose, we consider the following system of fractional differential Equations (FDE)

$$
D_{t}^{\alpha_{i}}\left(u_{i}(t)\right)=F_{i}\left(t, u_{1}, \cdots, u_{m}\right), t \geq 0,0<\alpha_{i} \leq 1,
$$

subject to the initial condition

$$
u_{i}(0)=c_{i}, i=1,2, \cdots, m,
$$

where $F_{i}(t), i=1,2, \cdots, m$, are known analytical functions.

Now the zeroth-order deformation equation of (6) is given by

$$
\begin{aligned}
& (1-p) L\left[\psi_{i}(t ; p)-u_{i 0}(t)\right] \\
& =p \hbar_{i} H_{i}(t) N_{i}\left[\psi_{i}(t ; p)\right], \\
& i=1,2, \cdots, m,
\end{aligned}
$$

Here $p \in[0,1]$ is an embedding parameter, $L_{i}$ are auxiliary linear operators satisfying $L_{i}(0)=0, \hbar_{i} \neq 0$ is an auxiliary parameter, $H_{i}(t) \neq 0$ is an auxiliary function, $u_{i 0}(t)$ is initial guess satisfy the initial condition (7) and $\psi_{i}(t ; p)$ are unknown functions. Obviously, when $p=0$, we have

$$
\psi_{i}(t, 0)=u_{i 0}(t), i=1,2, \cdots, m,
$$

when $p=1$, we have

$$
\psi_{i}(t, 1)=u_{i 0}(t), i=1,2, \cdots, m \text {. }
$$

Expanding $\quad \psi_{i . j}(t ; p), i=1,2, \cdots, m, j=1,2, \cdots, n \quad$ in Taylor's series with respect to $p$, we get

$$
\begin{aligned}
& \psi_{i}(t, p)=u_{i 0}(t)+\sum_{n=1}^{\infty} u_{i n}(t) p^{n}, \\
& i=1,2, \cdots, m,
\end{aligned}
$$

where

$$
u_{i n}(t)=\left.\frac{1}{n !} \frac{\partial^{n} \psi_{i}(t, p)}{\partial p^{n}}\right|_{p=0} .
$$

If the initial guesses $u_{i 0}(t)$, the auxiliary linear operator $L$ and the nonzero auxiliary parameter $\hbar_{i}$ may properly choose so that the power series (11) converges at $p=1$, one has

$$
u_{i}(t)=u_{i 0}(t)+\sum_{n=1}^{\infty} u_{i n}(t) .
$$

Define the vector

$$
\begin{aligned}
& \boldsymbol{u}_{i}(t)=\left\{u_{i 0}(t), u_{i 1}(t), \cdots, u_{i j}(t)\right\}, \\
& i=1,2, \cdots, j .
\end{aligned}
$$

Differentiating the zero-order deformation equation (8) $n$ times with respect to $p$, then setting $p=0$ and dividing them by $n$ ! and using (12), we have the so-called high-order deformation equations

$$
\begin{aligned}
& L_{i}\left[u_{i n}(t)-\chi_{n} u_{i(n-1)}(t)\right] \\
& =\hbar_{i} \Re_{i n}\left(\boldsymbol{u}_{i(n-1)}(t)\right), i=1,2, \cdots, m .
\end{aligned}
$$

Subject to the initial conditions

$$
u_{\text {in }}(0)=0, i=1,2, \cdots, m \text {, }
$$

where

$$
\begin{aligned}
& \mathfrak{R}_{i n}\left(\boldsymbol{u}_{i(n-1)}(t)\right)=\left.\frac{1}{(n-1) !} \frac{\partial^{n-1} N_{i}\left[\psi_{i}(t, p)\right]}{\partial p^{n-1}}\right|_{p=0}, \\
& i=1,2, \cdots, m, j=1,2, \cdots, n,
\end{aligned}
$$

and

$$
\chi_{n}=\left\{\begin{array}{ll}
0, & n \leq 1 \\
1, & n>1
\end{array}\right\}
$$

Called the $n$ th-order deformation equation.

Select the auxiliary linear operator $L_{i}=D_{t}^{\alpha_{i}}$, then the $n$ th-order deformation Equation (15) can be written in the form

$$
\begin{aligned}
& J^{\alpha_{i}} D^{\alpha_{i}}\left[u_{i n}(t)-\chi_{n} u_{i(n-1)}(t)\right] \\
& =\hbar_{i} J^{\alpha_{i}}\left[H_{i}(t) \Re_{i n}\left(\boldsymbol{u}_{i(n-1)}(t)\right)\right] .
\end{aligned}
$$


As fractional optimal differential equation has at least one solution, so for convergent homotopy series solution we can construct a kind of zeroth-order deformation equation as

$$
\begin{aligned}
u_{i n}(t) & =\chi_{n} u_{i(n-1)}(t) \\
& +\hbar_{i} J^{\alpha_{i}}\left[H_{i}(t) \Re_{i n}\left(\boldsymbol{u}_{i(n-1)}(t)\right)\right]
\end{aligned}
$$

And

$$
u_{i}(t)=u_{i 0}(t)+u_{i 1}(t)+u_{i 3}(t) \cdots
$$

In view of the homotopy analysis method presented above, if we select the auxiliary functions $H_{1}=H_{2}=$ $H_{3}=H_{4}=H_{5}=1$, we can construct the homotopy for presented model in fractional order as

$$
\begin{aligned}
\mathfrak{R}_{1 n}\left(\boldsymbol{P}_{(n-1)}(t)\right)= & D^{\alpha_{1}} P_{n-1}-b N+\beta_{1} \sum_{i=0}^{n-1} P_{i} L_{n-1-i} \\
& -\left(d_{1}+\mu\right) P_{n-1}+\tau Q_{n-1}, \\
\mathfrak{R}_{2 n}\left(\boldsymbol{L}_{(n-1)}(t)\right)= & D^{\alpha_{2}} L_{n-1}-\beta_{1} \sum_{i=0}^{n-1} P_{i} L_{n-1-i} \\
& +\beta_{2} \sum_{i=0}^{n-1} L_{i} S_{n-1-i}+\left(d_{1}+\mu\right) L_{n-1}, \\
& +\left(\gamma+d_{3}+\mu\right) S_{n-1}, \\
\mathfrak{R}_{3 n}\left(\boldsymbol{S}_{(n-1)}(t)\right)= & D^{\alpha_{3}} S_{n-1}-\beta_{2} \sum_{i=0}^{n-1} L_{i} S_{n-1-i} \\
\mathfrak{R}_{4 n}\left(\boldsymbol{Q}_{(n-1)}(t)\right)= & D^{\alpha_{4}} Q_{n-1}-\gamma S_{n-1} \\
& +\left(\gamma+d_{4}+\mu\right) Q_{n-1}, \\
\mathfrak{R}_{5 n}\left(\boldsymbol{N}_{(n-1)}(t)\right)= & D^{\alpha_{4}} N_{n-1}-(b-\mu) N_{n-1} \\
& +d_{1} N_{n-1}+d_{2} L_{n-1}+d_{3} S_{n-1}+d_{4} Q_{n-1}
\end{aligned}
$$

Consequently we have

$$
\begin{gathered}
P_{n}=\chi_{n} P_{n-1}+\hbar_{1} J^{\alpha_{1}}\left[\mathfrak{R}_{1 n}\left(\boldsymbol{P}_{(n-1)}(t)\right)\right], \\
L_{n}=\chi_{n} L_{n-1}+\hbar_{2} J^{\alpha_{2}}\left[\mathfrak{R}_{2 n}\left(\boldsymbol{L}_{(n-1)}(t)\right)\right], \\
S_{n}=\chi S_{n-1}+\hbar_{3} J^{\alpha_{3}}\left[\mathfrak{R}_{3 n}\left(\boldsymbol{S}_{(n-1)}(t)\right)\right], \\
Q_{n}=\chi_{n} Q_{n-1}+\hbar_{4} J^{\alpha_{4}}\left[\mathfrak{R}_{4 n}\left(\boldsymbol{Q}_{(n-1)}(t)\right)\right], \\
N_{n}=\chi_{n} N_{n-1}+\hbar_{5} J^{\alpha_{5}}\left[\mathfrak{R}_{5 n}\left(\boldsymbol{N}_{(n-1)}(t)\right)\right] .
\end{gathered}
$$

\section{Numerical Method and Simulation}

The system of Equations (1)-(5) with initial conditions were solved analytically by using homotopy analysis method and numerically using the classical Runge Kutta method in the case of integer derivative. For numerical results of the system of Equations (1)-(5) we use the following values of parameters,

$$
\begin{aligned}
& b=0.0045, \beta_{1}=0.014, \beta_{2}=0.014, \\
& \gamma=0.0165, \mu=0.0021, \tau=0, d_{1}=0.034, \\
& d_{2}=0.045, d_{3}=0.054, d_{4}=0.061
\end{aligned}
$$

and initial conditions

$$
c_{1}=153, c_{2}=55, c_{3}=79, c_{4}=68, c_{5}=355 \text {. }
$$

Figures 1-5 show the approximate solutions obtained using the HAM and the classical Runge-Kutta method of $P(t), L(t), S(t), Q(t)$ and $N(t)$ for $\alpha=1$. From the graphical result of these figures, it can be seen that the results obtained using the HAM match the results of the classical Runge-Kutta method very well. Figures 6-10 show the approximate solutions for $P(t), L(t), S(t)$, $Q(t)$ and $N(t)$ obtained for different values of $\alpha$ using the homotopy analysis method. From the numerical

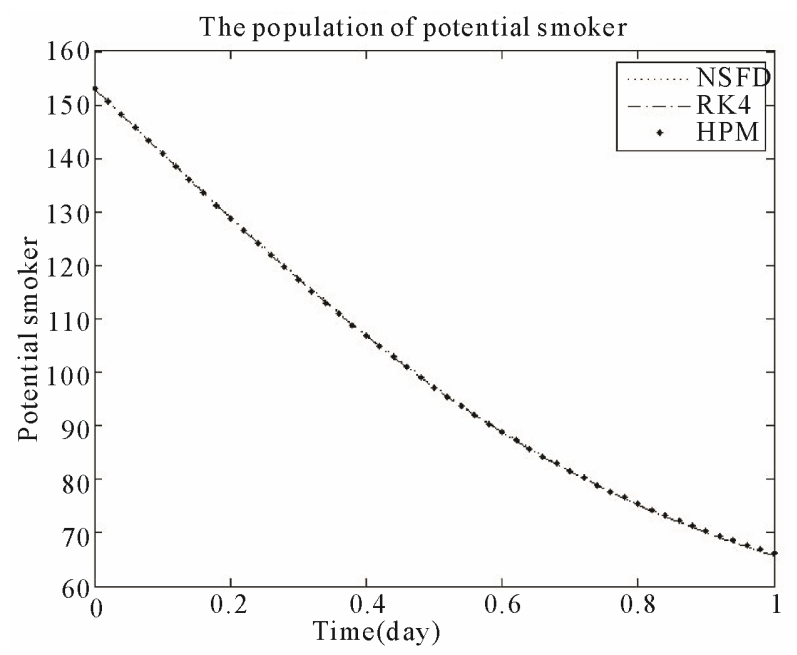

Figure 1. The plot shows the potential smokers individual.

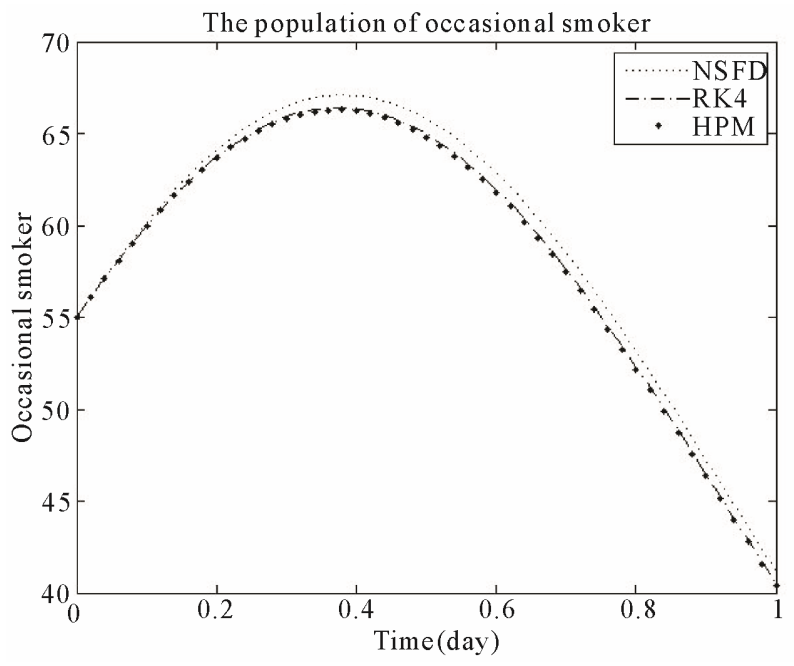

Figure 2. The plot shows the occasional smokers individual. 


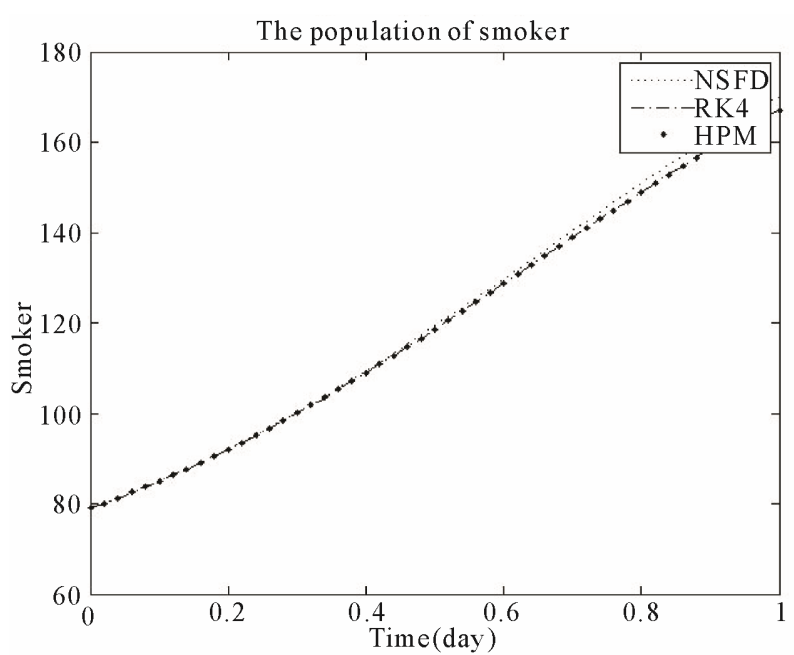

Figure 3. The plot shows the smokers individual.

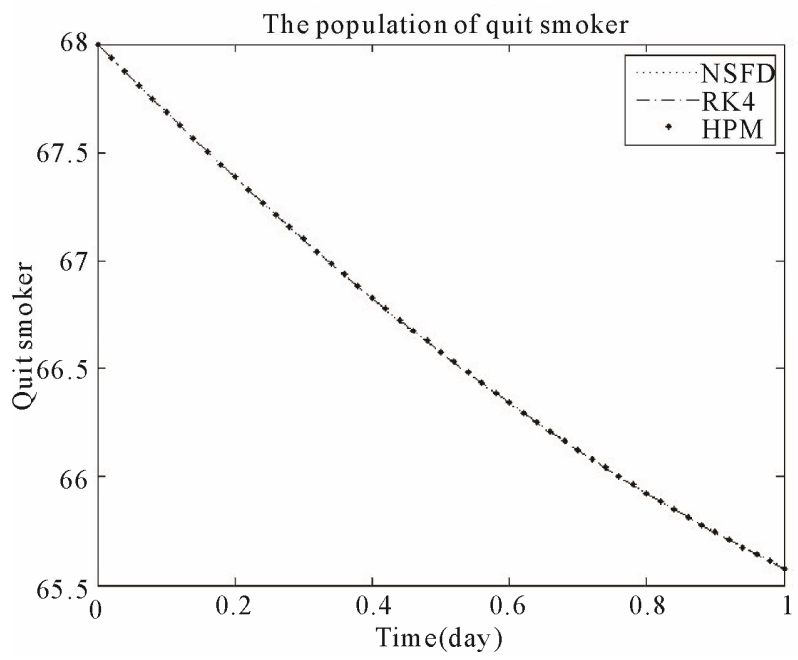

Figure 4. The plot shows the quit smokers individual.

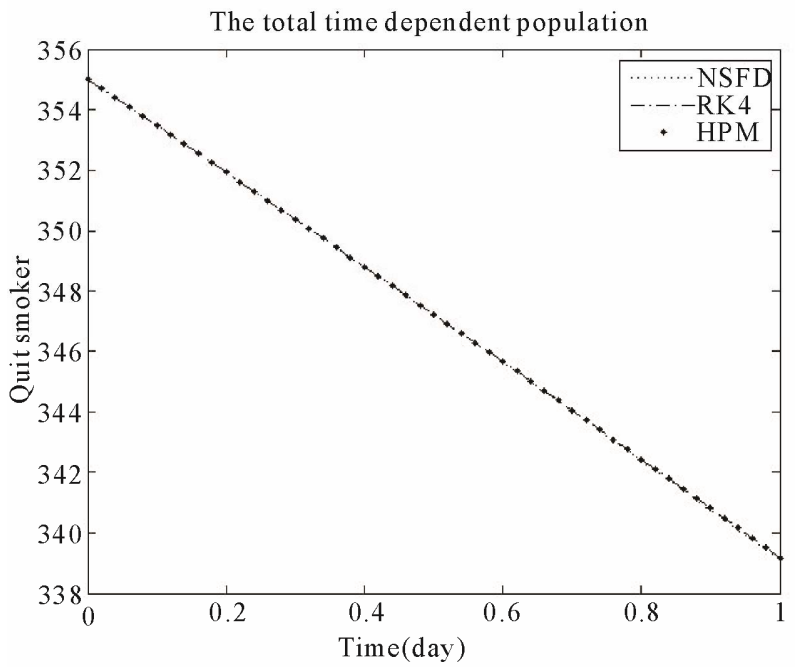

Figure 5. The plot shows the total population.

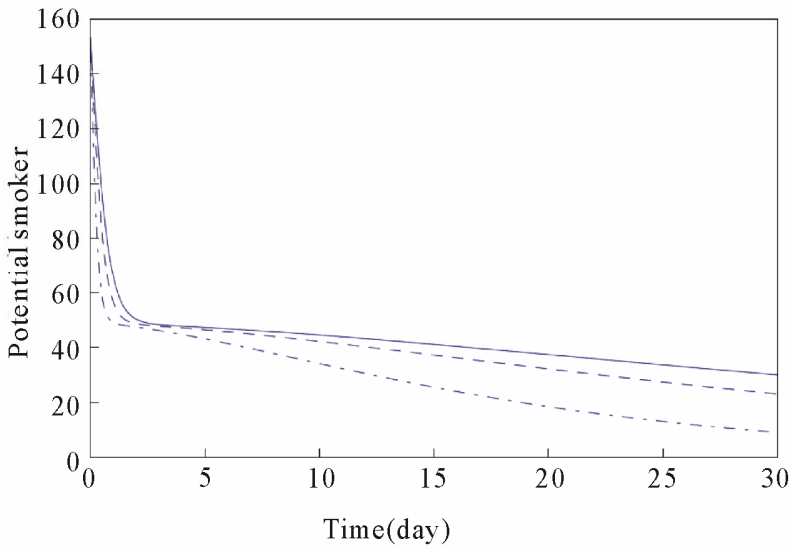

Figure 6. $P(t)$ versus $t$ : (solid line) $\alpha=1.0$, (dashed line) $\alpha=$ 0.95, (dot-dashed line) $\alpha=0.85$.

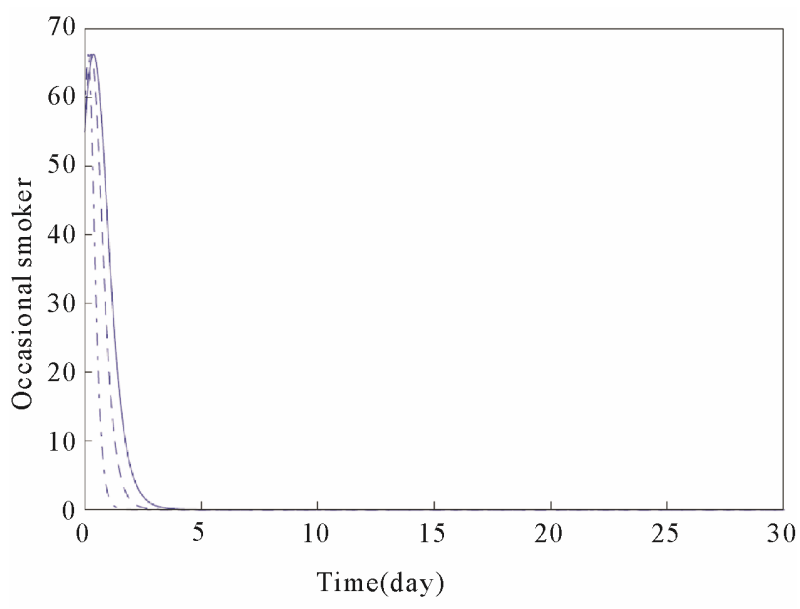

Figure 7. $L(t)$ versus $t$ : (solid line) $\alpha=1.0$, (dashed line) $\alpha=$ 0.95 , (dot-dashed line) $\alpha=0.85$.

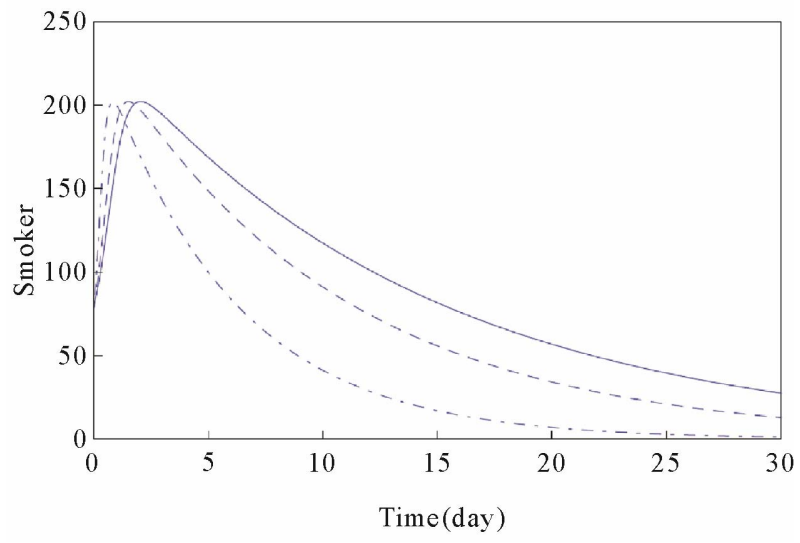

Figure 8. $S(t)$ versus $t$ : (solid line) $\alpha=1.0$, (dashed line) $\alpha=$ 0.95 , (dot-dashed line) $\alpha=0.85$.

results in these figures, it is clear that the approximate solutions depend continuously on the time-fractional derivative $\alpha$. 


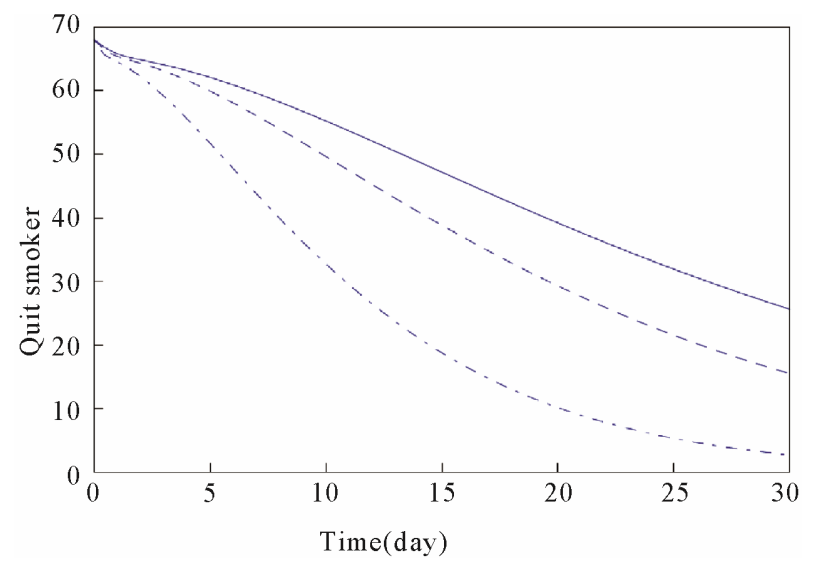

Figure 9. $Q(t)$ versus $t$ : (solid line) $\alpha=1.0$, (dashed line) $\alpha=$ 0.95, (dot-dashed line) $\alpha=0.85$.

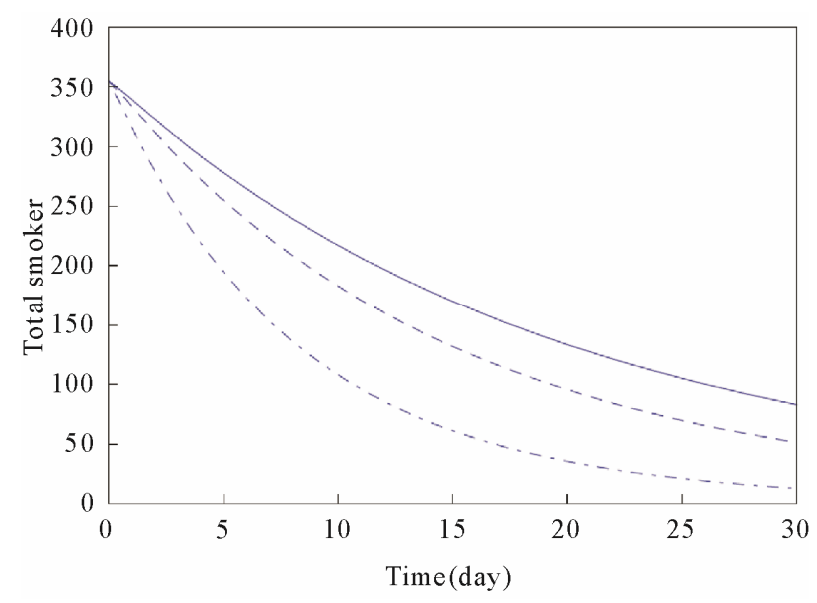

Figure 10. $N(t)$ versus $t$ : (solid line) $\alpha=1.0$, (dashed line) $\alpha=$ 0.95, (dot-dashed line) $\alpha=0.85$.

\section{Conclusion}

In this paper, we considered the giving up smoking model in fractional order. The homotopy analysis method (HAM) employed to compute an approximate and analytical solution of the model in fractional order. The obtained results are compaired with those obtained by forth order Runge-Kutta method and nonstandard numerical method in the integer case. Finally, we shown some numerical results.

\section{Acknowledgements}

The work of Dr. M. Ikhlaq Chohan was partially supported by the Business and Accounting Department $\mathrm{Al}$
Buraimi University College Al Buraimi, Oman.

\section{REFERENCES}

[1] I. Podlubny, "Fractional Differential Equations," Academic Press, London, 1999.

[2] L. Debnath, "Recent Applications of Fractional Calculus to Science and Engineering," International Journal of Mathematics and Mathematical Sciences, Vol. 54, 2003, pp. 3413-3442.

[3] S. Miller and B. Ross, “An Introduction to the Fractional Calculus and Fractional Differential Equations,” Willey, New York, 1993.

[4] G. Zaman, "Qualitative Behavior of Giving Up Smoking Models," Bulletin of the Malaysian Mathematical Sciences Society, Vol. 34, 2011, pp. 403-415.

[5] S. J. Liao, "Notes on the Homotopy Analysis Method: Some Definitions and Theorems," Communications in Nonlinear Science and Numerical Simulation, Vol. 14, No. 4, 2009, pp. 983-997. doi:10.1016/j.cnsns.2008.04.013

[6] S. J. Liao, "The Proposed Homotopy Analysis Technique for the Solution of Nonlinear Problems,” Ph.D. Thesis, Shanghai Jiao Tong University, Shanghai, 1992.

[7] M. Zurigat, S. Momani, Z. Odibat and A. Alawneh, “The Homotopy Analysis Method for Handling Systems of Fractional Differential Equations," Applied Mathematical Modelling, Vol. 34, No. 1, 2010, pp. 24-35.

doi:10.1016/j.apm.2009.03.024

[8] M. Zurigat, S. Momani and A. Alawneh, "Analytical Approximate Solutions of Systems of Fractional Algebraic-Differential Equations by Homotopy Analysis Method," Computers \& Mathematics with Applications, Vol. 59, No. 3, 2010, pp. 1227-1235. doi:10.1016/j.camwa.2009.07.002

[9] S. Momani and Z. Odibat, "Homotopy Perturbation Method for Nonlinearl Partial Differential Equations,” Physics Letters A, Vol. 365, No. 5-6, 2007, pp. 345-350. doi:10.1016/j.physleta.2007.01.046

[10] Z. Odibat, S. Momani and H. Xu, "A Reliable Algorithm of Homotopy Analysis Method for Solving Nonlinear Fractional Differential Equations,” Applied Mathematical Modelling, Vol. 34, No. 3, 2010, pp. 593-600. doi:10.1016/j.apm.2009.06.025

[11] S. Momani, V. S. Erturk and G. Zaman, "An Approximate Solution of a Giving Up Smoking Model in Fractional Order," Computers \& Mathematics with Applications, 2012, in press. 